\title{
The association between indwelling urinary catheter use in the elderly and urinary tract infection in acute care Susan E Hazelett* ${ }^{*}$, Margaret Tsai ${ }^{\dagger}$, Michele Gareri ${ }^{\dagger}$ and Kyle Allen ${ }^{\dagger}$
}

Address: 41 Arch St., room 212, Summa Health System, Akron, Ohio, 44304, USA

Email: Susan E Hazelett* - hazelets@summa-health.org; Margaret Tsai - Margaret.mt1@gmail.com; Michele Gareri - garerim@summahealth.org; Kyle Allen - allenk@summa-health.org

* Corresponding author †Equal contributors

Published: 12 October 2006

BMC Geriatrics 2006, 6:15 doi:10.1/86/147|-23/8-6-15
Received: 17 July 2006

Accepted: 12 October 2006

This article is available from: http://www.biomedcentral.com/I47I-23/8/6/15

(c) 2006 Hazelett et al; licensee BioMed Central Ltd.

This is an Open Access article distributed under the terms of the Creative Commons Attribution License (http://creativecommons.org/licenses/by/2.0), which permits unrestricted use, distribution, and reproduction in any medium, provided the original work is properly cited.

\begin{abstract}
Background: The use of indwelling urinary catheters (IUCs) is thought to be the most significant risk factor for developing nosocomial urinary tract infections (UTIs). However, it is unclear how many elderly patients have preexisting bacteriuria prior to IUC placement. The purpose of this study was to determine I) the frequency and appropriateness of IUC use in the Emergency Department (ED) in elderly patients admitted to our acute care hospital, 2) the percentage of elderly patients with an IUC who were discharged from the hospital with a diagnosis of UTI, 3) the percentage of patients with IUCs who were diagnosed and treated for UTI in the ED or who had admission bacteriuria $\geq 10^{5}$ organisms $/ \mathrm{ml}$ indicating preexisting UTI, and 4) the percentage of patients with no indication of UTI on admission who had inappropriately placed IUCs and subsequently were diagnosed with a UTI.
\end{abstract}

Methods: Retrospective chart review. Chi square used to test significance of differences in proportions.

Results: Seventy three percent of patients who received an IUC in the ED were elderly ( $\geq 65$ years old). During the study period, 277 elderly patients received an IUC prior to admission. Of these, 77 (28\%) were diagnosed with UTI during their hospitalization. Fifty three (69\%) of those diagnosed with a UTI by discharge either had the UTI diagnosed in the ED or had bacteriuria $\geq 10^{5}$ organisms/ $\mathrm{ml}$ prior to IUC placement. Of the 24 elderly patients who developed a catheter-associated UTI (i.e., $9 \%$ of the elderly population who received an IUC), II of the IUCs were placed inappropriately. Thus, $4 \%$ of elderly patients with no indication of UTI on admission who received an inappropriate IUC in the ED had a primary or secondary diagnosis of UTI by discharge. The overall rate of nosocomial UTI due to an inappropriately placed IUC was the same in males and females.

Conclusion: This study indicates that the strong association between IUC use and UTI may be partly explained by the high prevalence of preexisting UTI prior to IUC placement. Further prospective studies are needed to clarify the true risk vs benefit ratio for IUC use in acutely ill elderly patients. 


\section{Background}

Approximately 4 million people each year receive an indwelling urinary catheter (IUC) [1] and 5-20\% of hospitalized patients who receive an IUC will be diagnosed with a urinary tract infection (UTI) $[1,2]$. IUC use is thought to be the most significant risk factor for developing nosocomial UTIs, especially in acutely ill elderly patients [3-6]. Indeed, the leading category of nosocomial infections are UTIs [7], and $80 \%$ of these are associated with IUCs [1]. UTIs are associated with increased morbidity, mortality, length of stay, and costs [4,8-11]. Even with improvements in nursing care of the catherized patient and experimental redesign of catheters themselves, UTI remains a problem in the catherized patient $[1,10,12-15]$. It is important, therefore, to carefully weigh the risks vs benefits in patients for whom an IUC is being considered and to minimize the inappropriate use of IUCs.

The association between UTIs and IUCs in acutely ill patients is well documented. However, this association does not establish cause and effect and most studies that establish the association between IUC and UTI do not examine whether the UTI preceeded IUC placement. One study by Gardam et al [5] showed that 2 of 24 (8.3\%) consecutive patients with IUCs in the Emergency Department (ED) had a UTI prior to catheter insertion, and 5 of the 24 (20.8\%) developed a catheter-associated UTI during their hospital stay. Three of these 5 UTIs were associated with inappropriate IUC placement. However, this study was small, urine cultures were not obtained consistently, and it included patients of all ages.

The presence of a preexisting UTI may be more likely in elderly patients admitted to the hospital $[1,8]$. Previous studies have found that asymptomatic bacteriuria affects up to $50 \%$ of noninstitutionalized geriatric women and $30 \%$ of geriatric men [9]. Johansson et al [11] found that $38 \%$ of elderly patients with hip fractures had positive urine cultures on admission to the hospital. In addition, O'Donnell et al [16] point out that $20-50 \%$ of nursing home residents have asymptomatic bacteriuria, and this rises to $100 \%$ in patients with IUCs. Several factors predispose the elderly to UTIs including functional abnormalities (e.g., enlarged prostate gland, obstructions), chronic diseases (e.g., diabetes, cerebrovascular disease, and neurodegenerative diseases such as Parkinson's and multiple sclerosis), and certain medications.

The current study attempts to replicate the findings of Gardam et al [5] with a larger sample. In addition, this study focuses exclusively on elderly patients (i.e., those $\geq 65$ years old).

The purpose of this study was to 1) examine the frequency and appropriateness of IUC use in the ED in elderly patients admitted to our acute care hospital, 2) to determine the percentage of elderly patients with an IUC who were discharged from the hospital with a primary or secondary diagnosis of UTI, 3) to determine the percentage of patients with IUCs who were diagnosed and treated for UTI in the ED or who had admission bacteriuria $\geq 10^{5}$ organisms $/ \mathrm{ml}$ indicative of preexisting UTI, and 4) to determine the percentage of elderly patients with no indication of UTI on admission who had inappropriately placed IUCs and subsequently were diagnosed with a UTI.

\section{Methods}

This study used retrospective chart review of all patients admitted to our acute care hospital from the ED with an IUC from March 1-31, 2004. Per hospital policy, all catheters were placed using sterile technique. Data collected included the patient's age, diagnoses, results of urine cultures, and the reason for IUC use. The significance of differences in proportions was determined using Chi square. Permission to conduct this study was obtained from our institution's Institutional Review Board (IRB).

For the purposes of this study, the presence of a UTI on admission was defined as 1) an admission urine culture with $\geq 10^{5}$ organisms $/ \mathrm{ml}$ or 2 ) the diagnosis and treatment of UTI by the ED physician. Urine cultures positive only for urogenital flora were considered negative for UTI for this study.

Catheter appropriateness was determined using the criteria of Nickel [3]. IUCs were considered appropriate for surgery, accurate measurement of intake and output, urinary retention, urinary incontinence posing a risk to the patient, urinary obstruction, altered blood pressure or blood volume status requiring accurate urine measurement, urine measurement in an uncooperative patient, bladder irrigation for a urinary tract hemorrhage, and palliative care for the terminally ill. This information was obtained by chart review by Registered Nurses.

\section{Results \\ Reason for IUC placement in the elderly}

Between March $1^{\text {st }}$ and March 31 2004,1633 patients were admitted to our acute care hospital from the ED. Of these patients, a total of 379 patients received an IUC (figure 1). Of these, 277 (73\%) were patients $\geq 65$ years old. Significantly more elderly patients received and IUC than those $<65$ years old (30\%vs $12 \%$, respectively, $\mathrm{p}<.0001$ ). Overall, 139 (51\%) of the elderly had IUCs appropriately placed and 138 (49\%) had inappropriately placed IUCs. Table 1 shows the primary reason patients received an IUC as determined by chart review. It also shows the number of patients with an IUC for each reason who had a UTI at admission, who had a UTI diagnosed by dis- 
1633 patients treated in the ED and admitted to the hospital

$379(23 \%)$ patients received an IUC

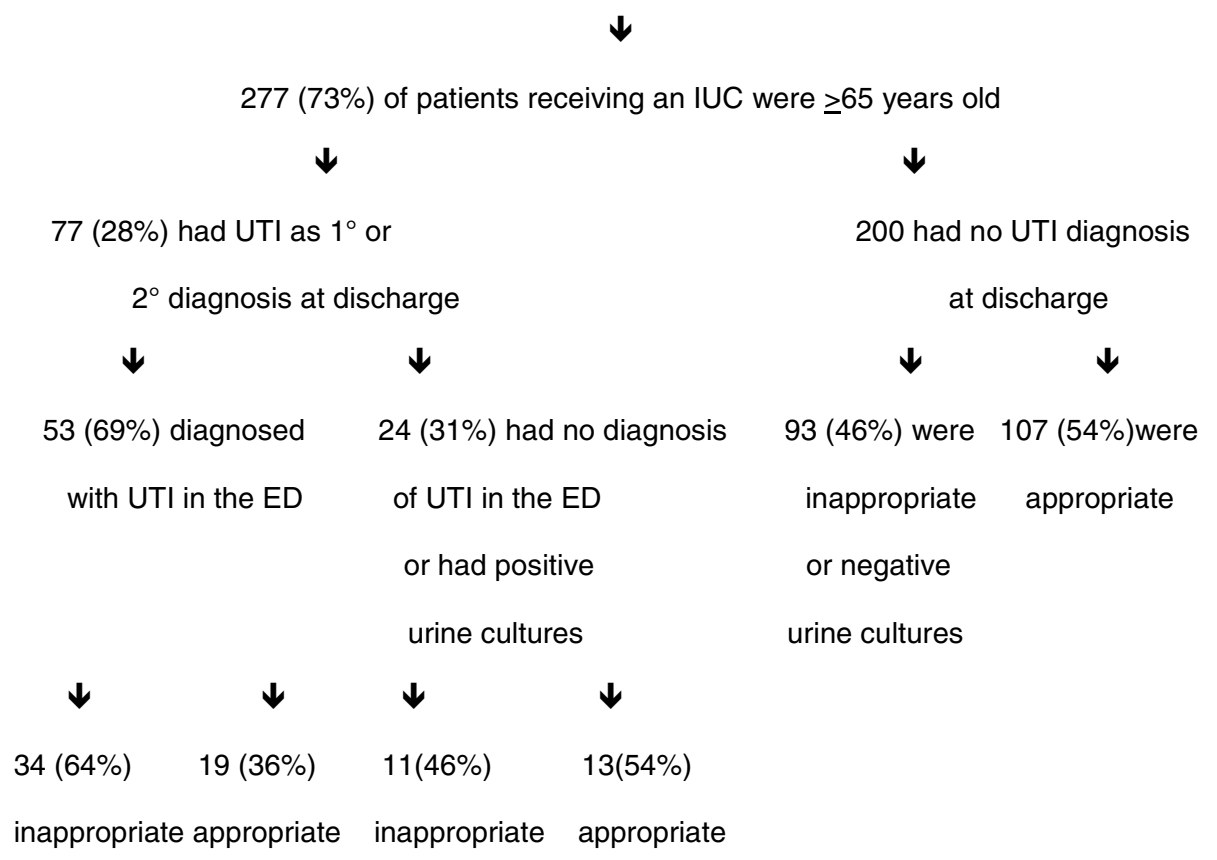

Figure I

Association between IUC use and UTI in an acutely ill elderly population.

charge, and the number who developed a nosocomial UTI.

\section{Percentage of catherized patients who developed a nosocomial UTI}

Seventy seven (28\%) of the 277 elderly patients who received an IUC had UTI as a primary or secondary diagnosis at discharge. These patients ranged in age from 65 to 101 years old and 32 (42\%) were from a nursing home. Only 57 of the 77 (74\%) had urine cultures performed, 40 of which were positive and 17 were negative. Four of the 77 were being treated for a UTI prior to arrival in the ED, 3 had urosepsis, 34 displayed clinical signs/symptoms of UTI, and 36 had asymptomatic UTIs. Significantly more patients with an IUC had a UTI diagnosed by discharge than those with no IUC $(28 \%$ vs $10 \%$, respectively, p < $.0001)$. Of the 77, 53 (69\%) were diagnosed and treated for UTI in the ED or had urine cultures showing $\geq 10^{5}$ organisms/ml, leaving 24 elderly patients without a UTI on admission who received an IUC and subsequently developed a UTI. In other words, 9\% of the elderly population who received an IUC developed a nosocomial UTI.
Association between nosocomial UTIs and inappropriately placed IUCs

Of the 24 elderly patients who developed a nosocomial UTI, 11 of the IUCs were determined to have been placed inappropriately (Table 2). Thus, $46 \%$ of the nosocomial UTIs were due to inappropriately placed IUCs. Overall 11 of the 277 (4\%) patients $\geq 65$ years old who received an IUC developed a nosocomial UTI associated with an inappropriately placed IUC.

Of the 200 elderly patients who received an IUC but were not diagnosed with a UTI during their hospital stay, 93 (46\%) of the IUCs were placed inappropriately, while 107 (54\%) were appropriately placed.

\section{Gender differences}

A significantly greater proportion of elderly patients receiving an IUC were female vs male (73\% vs $27 \%$ respectively, $\mathrm{p}<.0001$ ) (figure 2 ). Both groups, however, had similar rates of UTI as a primary or secondary diagnosis at discharge (27\% females, $29 \%$ males). Of those diagnosed with UTI by discharge, both groups also had similar 
Table I: Primary reason for indwelling urinary catheter placement

\begin{tabular}{|c|c|c|c|c|}
\hline Clinical Indication & $\begin{array}{l}\text { Number of patients with } \\
\text { IUC for this reason }\end{array}$ & $\begin{array}{l}\text { Number with IUC for this } \\
\text { reason who had UTI diagnosis } \\
\text { by discharge }\end{array}$ & $\begin{array}{l}\text { Number with IUC for this } \\
\text { reason who had UTI } \\
\text { diagnosis at admission }\end{array}$ & $\begin{array}{l}\text { Nosoc } \\
\text { omial } \\
\text { UTI }\end{array}$ \\
\hline Confusion & 42 & 21 & 18 & 3 \\
\hline Fractured Hip & 17 & I & 0 & 1 \\
\hline Intubated/IntensiveCareUnit/CriticalCareUnit admission & 36 & 6 & 4 & 3 \\
\hline Pneumonia & 4 & 0 & 0 & 0 \\
\hline Rectal bleed & 1 & 0 & 0 & 0 \\
\hline Intake\&Output & 7 & 5 & 3 & 2 \\
\hline Weakness & 4 & 2 & 2 & 0 \\
\hline Surgical case & 21 & 2 & I & 1 \\
\hline Dehydration & 6 & 3 & 2 & I \\
\hline Unresponsive/Loss of conciousness & 14 & 4 & 2 & 0 \\
\hline Short of breath & 4 & 1 & 0 & 1 \\
\hline Unable to ambulate & 9 & 4 & 2 & 1 \\
\hline Do Not Resucitate status & 2 & 0 & 0 & 0 \\
\hline Preexisting catheter & 3 & 3 & 2 & 1 \\
\hline Restrained & 2 & I & I & 0 \\
\hline Renal failure & 2 & I & 0 & 1 \\
\hline Urinary retention & 3 & I & 1 & 0 \\
\hline IntensiveCareUnit admission looked likely at admission & 4 & 0 & 0 & 0 \\
\hline Trauma & 4 & 0 & 0 & 0 \\
\hline Blood in urine & 3 & 0 & I & 0 \\
\hline Fall/syncope prior to arrival & 11 & 3 & 2 & 3 \\
\hline stroke/hemiplegia & 7 & I & 2 & 0 \\
\hline Abdominal pain & 7 & 4 & 3 & 1 \\
\hline Urinalysis/cultures & 13 & 5 & 4 & 1 \\
\hline CongestiveHeartFailure & 42 & 7 & I & 4 \\
\hline Cardiac catherization & 1 & 0 & 0 & 0 \\
\hline Gastrointestinal bleed & 2 & 0 & 0 & 0 \\
\hline Incontinent prior to arrival & 3 & 2 & 2 & 0 \\
\hline Cellulitis & 1 & 0 & 0 & 0 \\
\hline
\end{tabular}

rates of nosocomial UTI (29\% females, 36\% males). In females with nosocomial UTIs, IUC placement was inappropriate $50 \%$ of the time while in males with nosocomial UTIs, IUC placement was inappropriate only $25 \%$ of the time. Overall, $4 \%$ of all elderly females and $3 \%$ of all elderly males developed a nosocomial UTI after having an IUC placed inappropriately.

\section{Discussion}

IUCs are routinely cited as the primary cause of nosocomial infections [3-6]. On the other hand, previous studies have shown that decreasing IUC use is not associated with a decrease in UTIs [17]. The present study showed that $28 \%$ of elderly patients who received an IUC were diagnosed with a UTI during their hospitalization. These data support the strong association between IUC use and a diagnosis of UTI that has been shown in previous studies. However, closer examination of the clinical data reveals that $53(69 \%)$ of the patients who were diagnosed with UTI had either clinical signs/symptoms of UTI that were diagnosed and treated in the ED or they had an admission bacteriuria $\geq 10^{5}$ organisms $/ \mathrm{ml}$ indicative of a preexisting UTI. Thus, only $9 \%$, rather than $28 \%$, of elderly patients who received an IUC developed a nosocomial UTI. Thus, this study suggests that when preexisting UTIs are eliminated from the data, the association between IUC use and nosocomial UTI may be weaker than has been previously thought.

There is general agreement that the use of IUCs is appropriate in specific clinical situations. None-the-less, previous studies have shown that when using agreed upon definitions of appropriateness for IUC use, only about $50 \%$ of IUCs are used appropriately $[2,18]$. Our results support these previous findings by showing that in this elderly population with IUCs who developed a nosocomial UTI, only $54 \%$ of the IUCs were placed appropriately. However, this means that only 11 of the 277 (4\%) patients $\geq 65$ years old who received an IUC developed a nosocomial UTI associated with an inappropriately placed IUC. Thus, when IUC appropriateness is taken into consideration, the proportion of preventable nosocomial UTIs may also be smaller than previously thought.

In this study, female elderly patients were more susceptible to inappropriate IUC use than their male counterparts. Indeed, only two (25\%) male patients who developed a nosocomial UTI had an IUC placed inappropriately, while $8(50 \%)$ females who developed a nosocomial UTI had an inappropriately placed IUC. However, overall males and females were essentially equal in their rate of nosocomial UTI associated with an inappropriately placed IUC. 
Table 2: Reason for indwelling urinary catheters in patients who developed a nosocomial urinary tract infection by appropriateness $(n=24)$

\begin{tabular}{lc}
\hline Appropriate $(\mathbf{n}=\mathbf{~ I 3 )}$ & Number \\
\hline Diagnosis & 4 \\
\hline Congestive Heart Failure & $\mathrm{I}$ \\
Renal failure & 2 \\
Intake and output & 3 \\
Intensive Care Unit admission & $\mathrm{I}$ \\
Hip fracture & $\mathrm{I}$ \\
Surgical candidate & $\mathrm{I}$ \\
Urology patient (catheter prior to arrival) & \\
Inappropriate (n = II) & $\mathrm{I}$ \\
Urinalysis/cultures & $\mathrm{I}$ \\
Dehydration & 3 \\
Fall/syncope & $\mathrm{I}$ \\
Abdominal pain & 3 \\
Decreased mental status & $\mathrm{I}$ \\
Short of breath & $\mathrm{I}$ \\
Unable to ambulate & \\
\hline
\end{tabular}

The main limitation of this study was the use of retrospective chart review (with its inherent problems of missing data) to determine the appropriateness of IUC placement. As has been noted in previous studies, the reason for IUC placement was rarely explicitly stated in the chart and therefore had to be deduced from the available clinical information. However, the lack of documentation regarding the reason for IUC placement would only result in an underestimation of IUCs that were appropriate using the criteria established by Nickel [3]. For example, Nickel's criteria for appropriateness allows for catheter use for urine measurement in uncooperative patients. In our sample, the largest number of inappropriate IUCs was for confused patients, some of whom were undoubtedly uncooperative and therefore may have actually had an appropriately placed IUC. To ensure the most reliable interpretation of the reason for IUC placement from the chart review, Registered Nurses, one of whom was an ED nurse, were used as chart reviewers.

Rarely did it appear that there was a single reason for IUC placement. Indeed, it seemed in most cases that there were several patient characteristics contributing to the decision to use an IUC. For example, some patients were confused, dehydrated, and syncopal prior to arrival in the ED. Some had significant drops in their oxygen saturation levels when they were moved around in the bed. Some were in pain, immobile, and incontinent. While any one of these reasons alone may not meet the criteria for an appropriately placed IUC, we should consider the possibility that, taken together, the combination of clinical factors may tip the risk benefit scale. Perhaps appropriateness needs to be redefined on an individual basis when there is a constellation of factors that may contribute to IUC use.

Another limitation of this study is the fact that we did not record the duration of IUC use in relation to nosocomial UTI development. Numerous studies demonstrate that the risk of UTI increases as the duration of catherization increases $[9,12,18-20]$. Unfortunately, in this retrospective study we were unable to look at this relationship due to inconsistent documentation by nursing staff regarding the timing of IUC discontinuation.

The use of antibiotics might confound the results seen here. We did not examine correlations between antibiotic use and UTI in patients with IUCs due to the unreliable nature of pre-hospitalization medication histories. We know, however, that some patients who were diagnosed with UTI were being treated with antibiotics for various reasons prior to ED admission, some were treated with antibiotics in the ED, and some were treated with antibiotics on the nursing unit. Furthermore, we have no evidence to suggest that antibiotic use in our sample was substantially different from that which would be seen in any typical ED population. Thus, the effects of antibiotic use on the generalizability of our findings may be minimal.

A final limitation of this study may be the criteria used for a positive UTI (i.e., diagnosis and treatment of UTI by the $\mathrm{ED}$ physician or $\geq 10^{5}$ organisms $/ \mathrm{ml}$ in admission urine culture). While some authors define UTI simply by colony counts in urine cultures [21], others say that a positive diagnosis depends on the presence of UTI signs/symptoms and a positive urine culture [9]. Some authors define UTI as $\geq 10^{3}$ organisms/ml [23] and state that $>90 \%$ of these patients are asymptomatic. As stated by Nicolle [22], "neither a positive urine culture nor clinical presentation allows a diagnosis of symptomatic urinary infection to be made with a high degree of certainty" in the elderly. As with other studies, our definition is not optimal since UTI may be over-diagnosed and treated by ED physicians. Unfortunately, urine cultures are not routinely obtained when UTI is diagnosed and treated in the ED, making verification of the diagnosis difficult. However, there is also evidence from this study that UTI may be under-diagnosed in the ED as 18 patients had positive cultures but were not diagnosed with UTI in the ED. We chose $\geq 10^{5}$ organisms/ml or the presence of UTI symptoms prompting treatment in the ED as indicators of UTI which is in line with the Center for Disease Control and other national guidelines defining UTI $[24,25]$.

\section{Conclusion}

The results of this study indicate that IUC use following an emergency admission may not pose as high a risk for 
277 patients with IUCs $\geq 65$ years old

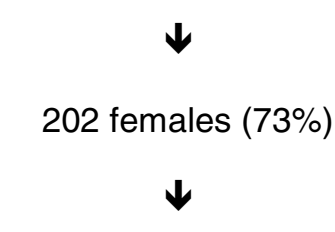

$55(27 \%)$ diagnosed with

UTI by discharge

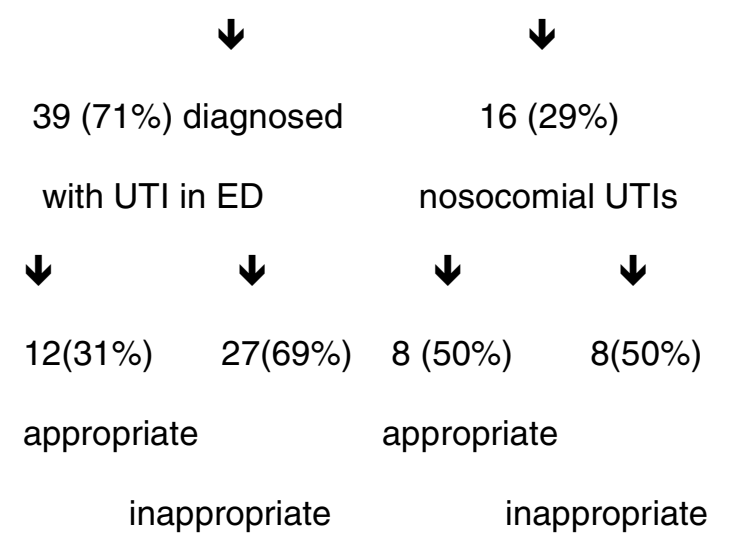

75 males $(27 \%)$

$22(29 \%)$ diagnosed with

UTI by discharge

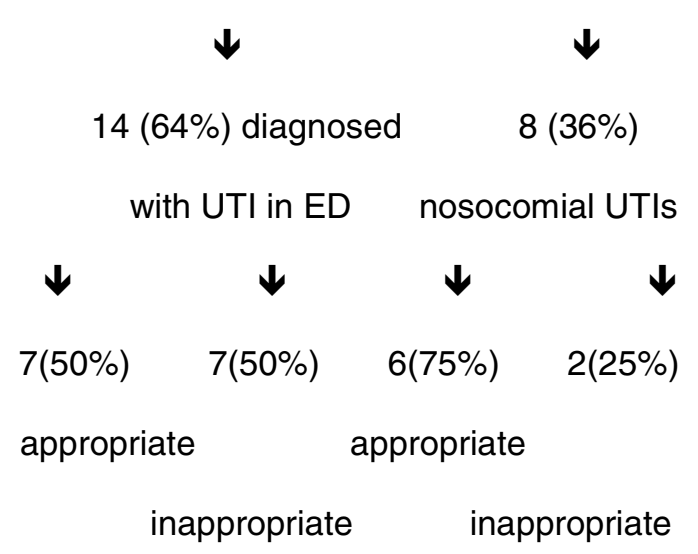

Figure 2

Gender differences in IUC outcomes.

nosocomial UTI as previously thought, nor are they as highly associated with preventable UTIs. These findings run contrary to conventional wisdom and to our own expectations. Further research using better documentation for the reason for IUC use and the duration of use is warranted. Furthermore, despite these findings there is still a sizeable percentage of patients who develop nosocomial UTIs after the inappropriate placement of an IUC. Given the morbidity and mortality associated with UTIs in acutely ill elderly patients, it is still reasonable to make every effort to limit the use of IUCs to only those patients for whom an IUC is appropriate. Finally, the indiscriminant use of IUCs in acute care hospitals should still be avoided in the elderly due to the associated increased risk of functional impairment, falls, and immobility. Further research is needed to clarify the relationship between IUC use and preventable UTIs so that clinicians can more accurately assess the risks compared to the benefits of IUC use.

\section{Competing interests}

The author(s) declare that they have no competing interests.

\section{Authors' contributions}

SH contributed to the study design, data collection, data analysis, and manuscript preparation. MT contributed to the study design, data collection, and manuscript preparation. MG contributed to study design, data collection, and manuscript preparation. KA conceived the study and contributed to the study design and manuscript preparation. All authors read and approved the final manuscript.

\section{Acknowledgements}

None

Funding for Susan E Hazelett, Margaret Tsai, Michele Gareri, Kyle Allen was provided by the Health Services Research and Education Institute with funds from the Summa Health System Foundation.

\section{References}

I. Wong ES: Guideline for prevention of catheter-associated urinary tract infections. CDC [http://www.cdc.gov/ncidod/dhqp/ gl catheter assoc.html].

2. Gokula RR, Hickner JA, Smith MA: Inappropriate use of urinary catheters in elderly patients at a Midwestern community teaching hospital. American Journal of Infection Control 2004, 32(4): 196-99.

3. Nickel JC: Catheter-associated urinary tract infection:new perspectives on problems. Canadian Journal of Infect Control I 99I, 6(2):38-42. 
4. Stamm WE: Scientific and clinical challenges in the management of urinary tract infections. The American Journal of Medicine 2002, II 3(IA): Is-4s.

5. Gardam MA, Amihod B, Orenstein P, Consolacion N, Miller M: Overutilization of indwelling urinary catheters and the development of nosocomial urinary tract infections. Clinical Performance and Quality Health Care 1998, 6:99-102.

6. Brosnahan J, Jull A, Tracy C: Types of urethral catheters for management of short-term voiding problems in hospitalized adults. Cochrane Database System Review 2004:CD0040I3.

7. Kalsi J, Arya M, Wilson P, Mundy A: Hospital-acquired urinary tract infection. International Journal of Clinical Practice 2003, 57(5):388-9|

8. Tal S, Guller V, Levi S, Bardenstein R, Berger D, Gurevich I, Gurevich $A$ : Profile and prognosis of febrile elderly patients with bacteremic urinary tract infection. Journal of Infection 2005, 50(4):296-305.

9. Foxman B: Epidemiology of urinary tract infections: incidence, morbidity, and economic costs. Disease Monthly 2003, 49(2):53-70

10. Lai KK, Fontecchio SA: Use of silver-hydrogel urinary catheters on the incidence of catheter-associated urinary tract infections in hospitalized patients. American Journal of Infection Control 2002, 30(4):22I-5.

II. Johansson I, Athlin E, Frykholm L, Bolinder H, Larsson G: Intermittent versus indwelling catheters for older patients with hip fractures. Journal of Clinical Nursing 2002, I I (5):65I-6.

12. Huang WC, Wann SR, Lin SL, Kunin CM, Kung MH, Lin CH, Hsu CW Liu CP, Lee SS, Liu YC, Lai KH, Lin TW: Catheter-associated urinary tract infections in intensive care units can be reduced by prompting physicians to remove unnecessary catheters. Infection Control Hospital Epidemiology 2004, 25( I I):974-8.

13. Misset B, Timsit JF, Dumay MF, Garrouste M, Chalfine A, Flouriot I, Goldstein $\mathrm{F}$, Carlet J: A continuous quality-improvement program reduces nosocomial infection rates in the ICU. Intensive Care Medicine 2004, 30(3):395-400.

14. Reiche T, Lisby G, Jorgensen S, Christensen AB, Nordling J: A prospective, controlled, randomized study of the effect of a slow-release silver device on the frequency of urinary tract infection in newly catheterized patients. BJU Int 2000, 85(I):54-9.

15. Rosenthal VD, Guzman S, Safdar N: Effect of education and performance feedback on rates of catheter-associated urinary tract infection in intensive care units in Argentina. Infection Control Hospital Epidemiology 2004, 25(I):47-50.

16. O'Donnell JA, Hofmann MT: Urinary tract infections. How to manage nursing home patients with or without chronic catheterization. Geriatrics 2002, 57(5):45,49-52. 55-6

17. Zimakoff J, Stickler DJ, Pontoppindan B, Larson SO: Bladder management and urinary tract infections in Danish hospitals, nursing homes, and home care: a national prevalence study. Infection Control Hospital Epidemiology 1996, 17:215-221.

18. Foxman B: Epidemiology of urinary tract infections: incidence, morbidity, and economic costs. The American Journal of Medicine 2002, II3(IA):5S-I3S.

19. Tissot E, Limat S, Cornette C, Capellier G: Risk factors for catheter-associated bacteriuria in a medical intensive care unit. European Journal of Clinical Microbiology and Infectious Diseases 200I, 20(4):260-2.

20. Leone M, Albanese J, Garnier F, Sapin C, Barrau K, Bimar M, Martin $C$ : Risk factors of nosocomial catheter-associated urinary tract infections in a polyvalent intersive care unit. Intensive Care Medicine 2003, 29(I 2):234I.

21. Tambyah PA, Maki DG: Catheter-associated urinary tract infection is rarely symptomatic: a prospective study of $I, 497$ catheterized patients. Archives of Internal Medicine 2000, I 60(5):678-82.

22. Nicolle LE: Urinary infections in the elderly: symptomatic of asymptomatic? International Journal of Antimicrobial Agents 1999, I I(3-4):265-8.

23. Tambyah PA, Maki DG: The relationship between pyuria and infection in patients with indwelling urinary catheters: a prospective study of $76 \mathrm{I}$ patients. Archives of Internal Medicine 2000, 160(5):673-7.
24. Organization and Implementation of Infection Control Programs [http://www.cdc.gov/ncidod/dhqp/pdf/nnis/NosInfDefini tions.pdf]

25. FamilyPractice.com [http://www.familypractice.com/referencereferencesframe.htm?main=/references/ABFPGuides/Urinary/ UTI flow.htm]

\section{Pre-publication history}

The pre-publication history for this paper can be accessed here:

http://www.biomedcentral.com/1471-2318/6/15/prepub
Publish with Biomed Central and every scientist can read your work free of charge

"BioMed Central will be the most significant development for disseminating the results of biomedical research in our lifetime. "

Sir Paul Nurse, Cancer Research UK

Your research papers will be:

- available free of charge to the entire biomedical community

- peer reviewed and published immediately upon acceptance

- cited in PubMed and archived on PubMed Central

- yours - you keep the copyright

Submit your manuscript here:

http://www.biomedcentral.com/info/publishing_adv.asp
BioMedcentral 